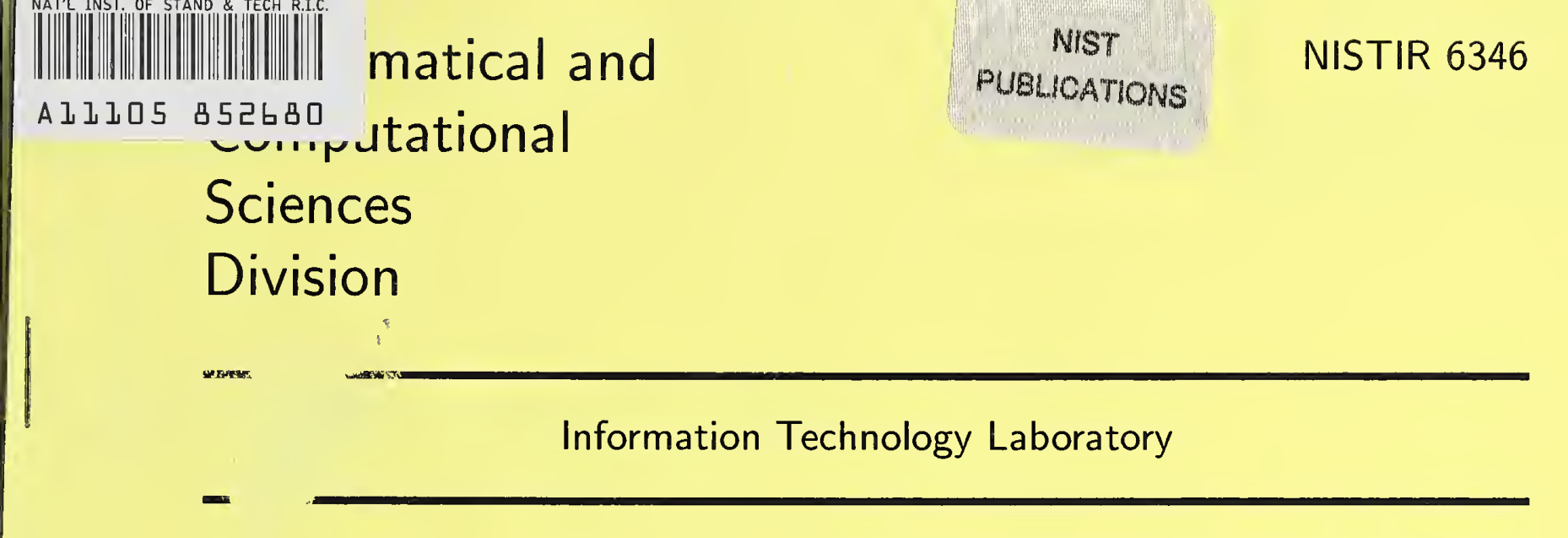

\title{
Triangulation-Based L1-fitting of Terrain Surfaces
}

\section{J. Bernal and C. Witzgall}

June 1999

\section{U.S. DEPARTMENT OF COMMERCE \\ Technology Administration \\ National Institute of Standards and Technology \\ Gaithersburg, MD 20899}

UC

100

.056

110.6340

1999 



\section{Triangulation-based $L_{1}$-fitting of Terrain Surfaces}

\section{Javier Bernal Christoph Witzgall}

U.S. DEPARTMENT OF COMMERCE Technology Administration National Institute of Standards and Technology

Mathematical and Computational Sciences Division

Gaithersburg, MD 20899, U.S.A ${ }^{1}$

${ }^{1}$ Partially supported by

DARPA/TEC MIPR 97-5039

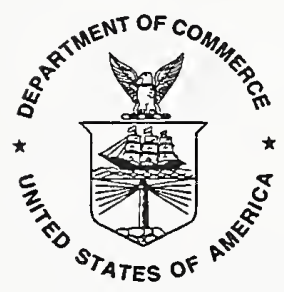

U.S. DEPARTMENT OF COMMERCE William M. Daley, Secretary

TECHNOLOGY ADMINISTRATION Gary R. Bachula, Acting Under Secretary for Technology

NATIONAL INSTITUTE OF STANDARDS

Raymond G. Kammer, Director 



\title{
Triangulation-based $L_{1}$-fitting of Terrain Surfaces
}

\author{
Javier Bernal and Christoph Witzgall \\ National Institute of Standards and Technology (NIST), Gaithersburg, MD 20899, U. S. A. ${ }^{1}$
}

${ }^{1}$ Partially supported by DARPA/TEC MIPR 97-5039 

Abstract. Given a planar triangulation, the goal is to select elevations at its vertices so that resulting piecewise-linear triangulated surface approximates specified elevations using the $L_{1}$-norm as the primary measure-of-fit. Several suboptimal algorithms relating to that problem have been devised and implemented, and are described here, as part of TIN prototype software for terrain modeling in the context of distributed simulation as well as elevation data editing. $L_{1}$-approximation acts as a median filter and thus provides advantages for "bare earth" representation. For added flexibility, a "sign-oriented scaling" scheme has been developed, which generalizes standard norm-based measures-of-fit. $L_{1}$-approximation is either tackled directly or by iterating suitably weighted $L_{2}$ approximations. Numerical experiments are reported.

Key words. bare earth, distributed simulation, $L_{1}$-approximation, $L_{2}$-approximation, linear programming, median filter, terrain surface modeling, TIN, triangulation

\section{Introduction}

"Triangulated irregular networks (TINs)" are playing an increasingly prominent role in modeling terrain surfaces for purposes such as animation, distributed simulation, and geodetic volumetrics (e.g. [3]). Typically, there is a rectangular map area together with a set $R$ of location points $p=\left(\hat{x}_{p}, \hat{y}_{p}\right)$ on that map each with elevation $\hat{z}_{p}$. Terrain is to be modeled as a surface based on those elevation data.

A triangulated irregular network is a planar triangulation which arises as the footprint of a triangulated surface $z=z(x, y)$, that is, a piecewise linear surface consisting of flat triangles joined continuously together in space. That surface is referred to as TIN-surface. Under triangulation, we understand any covering of the map by triangles such that the triangle interiors do not overlap and any two triangle edges which meet in more than one point are identical.

Typically, the vertices of the triangulation have previously been selected as critical points based on "greedy" criteria. We denote the set of those vertices by $S$. The TIN surface $z=z(x, y)$ is uniquely determined by the elevations $z_{s}$ at the critical points $s=\left(x_{s}, y_{s}\right) \in S$ and the footprint triangulation, the TIN: Indeed, the elevations at the three corners of any triangle in the TIN uniquely determine a plane through the corresponding elevation points and, therefore, a triangle in space.

The object then is to approximate given elevation data by a TIN surface - as accurately as possible with the number of triangles in the TIN not exceeding a prescribed upper limit, the "budget". Such restrictions are necessary, for instance, to meet the high speed requirements of real-time visualization. 
Generally, there are two aspects of this optimization. The first is the selection of the TIN itself, that is, of the critical points and their triangulation. The second is to assign elevations at those critical points so as to optinize some measure-of-fit to the given elevations while keeping the triangulation fixed. This work is concerned with that second aspect of optimization.

There are different measures-of-fit in general use to assess the accuracy of such an approximation. Most are based on the notion of a residual at each given elevation location $p=\left(\hat{x}_{p}, \hat{y}_{p}\right) \in R$,

$$
r_{p}=\hat{z}_{p}-\bar{z}_{p}=\hat{z}_{p}-z\left(\hat{x}_{p}, \hat{y}_{p}\right)
$$

where $\bar{z}_{p}$ denotes the elevation $z\left(\hat{x}_{p}, \hat{y}_{p}\right)$ which the TIN surface assumes at location $\left(\hat{x}_{p}, \hat{y}_{p}\right)$.

Two commonly considered measures-of-fit are the maximum deviation

$$
\operatorname{MAX}=\max _{p \in R}\left|r_{p}\right|
$$

and the root mean square

$$
\mathrm{RMS}=\sqrt{\frac{1}{|R|} \sum_{p \in R} r_{p}^{2}},
$$

where $|R|$ is the number of elevation locations. Both quantities relate to norms of vectors. MAX is an instance of the

$$
L_{\infty}-\text { norm }
$$

also called Chebychev norm. RMS is a scaled version of the

$$
L_{2}-\text { norm }
$$

or least-squares norm.

There is, however, a problem with $L_{2^{-}}$and, in particular, with $L_{\infty}$-norms, in that outliers among the elevation data caused by terrain artifacts such as rocks, single trees and bushes, as well as processing artifacts, distort the shape of the TIN surface - make it conform less to bare earth -, during the process of selecting elevations at critical points. In some approaches, the selection of a norm may also affect the construction of the TIN itself.

Those drawbacks of the commonly considered norms have led the authors to consider the

$$
L_{1}-\text { norm }
$$

as a measure-of-fit for terrain surface approximation because it is more robust with respect to artifacts, and may even be used for their detection (see [4]). The $L_{1}$-norm is defined as 
the sum of the absolute values of the residuals. One might actually prefer the mean absolute deviations

$$
\mathrm{MAD}=\frac{1}{|R|} \sum_{p \in R}\left|r_{p}\right| .
$$

Note that the elevation $z=z(x, y)$ of the TIN surface at any map location $(x, y)$ is a linear function of the critical elevations $z_{s}, s \in S$. Consequently, the residuals $r_{p}, p \in R$ are also linear functions of the critical elevations. For this reason, the $L_{1}$-approximation problem is a linear programming problem, and the $L_{2}$-approximation problem is a linear least squares problem. The connection between $L_{1}$-approximation and linear programming is well established (e.g.[1]).

More precisely, $z=z(x, y)$ is a linear combination of three elevations

$$
z_{s_{1}}, z_{s_{2}}, z_{s_{3}}
$$

which belong to vertices $s_{1}, s_{2}, s_{3}$ of a TIN triangle containing location $(x, y)$. The coefficients of that linear combination,

$$
z(x, y)=\lambda_{1} z_{s_{1}}+\lambda_{2} z_{s_{2}}+\lambda_{3} z_{s_{3}}
$$

are the barycentric coordinates of location $(x, y)$ with respect to $s_{i}=\left(x_{s_{i}}, y_{s_{i}}\right), i=1,2,3$ and are defined by

$$
\begin{aligned}
& x=\lambda_{1} x_{s_{1}}+\lambda_{2} x_{s_{2}}+\lambda_{3} x_{s_{3}} \\
& y=\lambda_{1} y_{s_{1}}+\lambda_{2} y_{s_{2}}+\lambda_{3} y_{s_{3}} \\
& 1=\lambda_{1}+\lambda_{2}+\lambda_{3} .
\end{aligned}
$$

The barycentric coordinates assume values between 0 and 1 since the location $(x, y)$ lies in the triangle in question. The barycentric coordinates play a major role in approximation by triangulated surfaces.

While linear programming problems are well understood and excellent solution methods exist even for large numbers of variables, the $L_{1}$-approximation problem considered here typically exceeds the size that can be handled by those methods. Suboptimal solution methods were, therefore, developed. Straight $L_{2}$-approximation, however, can be solved fully optimally on an iterative basis described below.

One method of elevation optimization with respect to a specific measure-of-fit is

$$
\text { vertex - based elevation optimization. }
$$

With any critical point - vertex in the TIN -, we associate the union of all triangles in the triangulation which contain that vertex, calling it the star of that critical point. The star is a 
simply-comnected polygon bounded by the edges between adjacent "neighbor" vertices of the critical point that centers the star.

We will require the triangulation to be

$$
\text { star-complete, }
$$

that is, every star in the triangulation contains at least one elevation location $\left(\hat{x}_{p}, \hat{y}_{p}\right), p \in R$ in its interior. Without this condition, the elevation at the center of an empty star could be chosen arbitrarily without affecting any of the norm-based measures-of-fit considered here. In that sense, the approximation problem would be ill-defined.

A single step of a vertex-based iteration then consists of adjusting the elevation at the center of a star so as to ninimize the norm of choice of the residuals in the interior of the star while keeping the elevations at all other vertices fixed. Each vertex elevation is adjusted in turn in a pass through all critical points. Such a pass is then repeated iteratively until the resulting improvements become acceptably small.

For $L_{1}$ - and $L_{2}$-norms, the basic vertex-based optimization step can be executed readily in closed form. In the $L_{2}$ case, a convex quadratic of one variable has to be minimized. In the $L_{1}$ case, minimization of a piecewise linear convex function of one variable is required. This task is equivalent to determining a suitably weighted median of the breakpoints.

In the case of the $L_{2}$-norm, furthermore, it can be shown that the vertex-based iterative procedure converges to the true optimum (e.g. [2]). That, however, does not hold in the case of the $L_{1}$-norm. For this reason, two additional algorithms for $L_{1}$-optimization have been developed in order to improve the degree of optimality that can be achieved.

The first of those algorithms is

$$
\text { edge-based elevation optimization, }
$$

where the basic step is to $L_{1}$-optimize the elevations at the two end-vertices of a triangulation edge simultaneously while keeping the elevations at all other vertices of the triangulation fixed. That local optimization problem turns out to be a special linear programming problem, and a special algorithm has been designed to solve it.

The second algorithm utilizes the fact that, for $p \in R$,

$$
\frac{r_{p}^{2}}{\left|r_{p}\right|}=\frac{\left(\hat{z}_{p}-\bar{z}_{p}\right)^{2}}{\left|\hat{z}_{p}-\bar{z}_{p}\right|}=\left|\hat{z}_{p}-\bar{z}_{p}\right|=\left|r_{p}\right| \text {, }
$$

to formulate a

$$
\text { residual - weighted } L_{2}-\text { procedure }
$$

for $L_{1}$-approximation. Roughly speaking, we start the $L_{2}$-approximation with equal weights, and adjust these weights as we proceed on the basis of observed residuals. The challenge here 
is to handle the cases of very small residuals which result in very large weights. There is also the question how far to iterate the $L_{2}$ procedure before updating the weights. Our current approach is to adjust weights after one $L_{2}$-pass through the vertices of the triangulation.

Both algorithms are still suboptimal and, as the numerical results presented in this report show, best results so far have been achieved by alternating both algorithms. We know that these results are suboptimal, because it is well known that, for any fully optimal solution of any $L_{1}$ approximation problem, the number of

\section{sharp}

elevation location points $\left(\hat{x}_{p}, \hat{y}_{p}\right) \in R$, that is, points for which $\hat{z}_{p}=\bar{z}_{p}$, and which are thus represented exactly by the TIN surface, essentially equals the number of degrees of freedom provided by the optimization parameters. Here that number is the number $\left|S \backslash S^{\prime}\right|$ of nonfixed critical points. For our best results, the number of sharp points does not quite reach that goal.

The algorithms and routines described here were devised and developed in the context of particular applications to terrain modeling. For these applications, the bulk of the elevation data points were provided as regular grid points with square unit cells covering a rectangular map exactly. A set $S^{\prime}$ of optional additional elevation points - "feature points" - were also specified with the proviso that they be included into the set of critical points $S$ and that their given elevations are not to be changed. If such a feature point happens to be located on the grid, then its given elevation takes precedence over the specified grid elevation. Furthermore, all critical points other than feature points had to be grid points. This ensured that all corresponding stars were complete, as defined above, because their respective center vertices are elevation points. In addition, the specified grid elevations at such critical points provided good initial values for iterations.

For the purpose of identifying terrain artifacts, it is useful to generalize $L_{1}$ approximation by scaling residuals differently depending on their sign. Our software thus includes an option to assign scale factors $u>0$ if $r_{p}>0$ and $v>0$ if $r_{p}<0$. We call that option

$$
\text { sign-oriented scaling. }
$$

If, for instance, the object is to identify artifacts above bare earth, then one would scale positive residuals lower than negative residuals. That would reduce the influence of artifacts above bare earth on the construction of the TIN surface. Those artifacts will then show up more clearly if the data are compared to the latter. Sign-oriented scaling can be specified for any norm.

The algorithms reported here are as follows. For quantities not defined earlier, we ask the reader to refer to the detailed descriptions provided later.

$$
\operatorname{L} 2 \mathrm{FIT}\left(R, S, S^{\prime}, T, F, Z, \hat{Z}, n, \text { tol }\right)
$$


is a vertex-based iterative procedure for $L_{2}$-approximation. It is known to actually converge to the optimum. It has been in use as a post-optimization option in the prototype NIST TIN package for several years.

$$
\operatorname{L1FIT1}\left(R, S, S^{\prime}, T, F, Z, \hat{Z}, n, \text { tol, npmax }, u, v\right)
$$

is a vertex-based iterative procedure for $L_{1}$-approximation featuring sign-oriented scaling. It is suboptimal. It was the first $L_{1}$ procedure to be considered.

$$
\text { L1FIT2 }\left(R, S, S^{\prime}, T, F, E, Z, \hat{Z}, n, \text { tol, npmax }, u, v\right)
$$

is an edge-based iterative procedure for $L_{1}$-approximation featuring sign-oriented scaling. It is suboptimal but achieves results that are closer to optimality than the vertex-based version. It was the second $L_{1}$ procedure to be considered.

$$
\operatorname{L1FIT3}\left(R, S, S^{\prime}, T, F, Z, \hat{Z}, n, \text { tol }, \text { npmax }, u, v, w s c l\right)
$$

aims to achieve an $L_{1}$ optimum by iterating a residual-weighted vertex-based $L_{2}$ procedure. Sign-oriented scaling is included. The procedure is still suboptimal but by itself achieves a higher degree of optimality than the previous procedure.

Comparative numerical results are reported for $L_{1}$-approximation of a representative set of terrain elevation data without feature points and without sign-oriented scaling. As was mentioned earlier, best results were found when procedures L1FIT2 and L1FIT3 were alternated. We feel that those results are close to optimal, particularly, because the number 19558 of sharp points approaches the number 20000 of critical points.

\section{Numerical results}

The procedures presented here for computing approximate $L_{1}$ solutions have been implemented at NIST. They were applied to a terrain data set consisting of a regular grid of $229,761(521 \times 441)$ points with square $25 \times 25 \mathrm{~m}$ unit cells covering a rectangular $11 \times 13 \mathrm{~km}$ map. A triangulation of 20,000 critical points - selected from among the grid points and covering the map - was also given. None of the critical points were feature points with fixed elevations, so $S^{\prime}=\emptyset$. Sign-oriented scaling was not used, so $u=v=1$.

Table 2.1, Table 2.2, and Table 2.3 show some numerical results obtained when the vertexbased procedure, the edge-based procedure, and the $L_{2}$ with weights procedure, respectively, were used on the test problem. Table 2.4 shows some numerical results obtained when the edge-based procedure was used on the test problem after 300 passes through the vertices by the $L_{2}$ with weights procedure. In all cases, where it applies, variable tol was set to $6.058 \mathrm{e}-04$, and variable $w s c l$ to 1.05 . Given an integer $i, 1 \leq i \leq n$, where it applies, $E(i)$ was set to the 


\begin{tabular}{||r|ccccc||}
\hline \hline pass & CPUsec & objective & residual tolerance & MAX residual & \# sharp points \\
\hline 1 & 121.9 & 2508140.342 & 158.750 & 14976 \\
2 & 121.6 & 2466989.055 & 158.750 & 15015 \\
5 & 121.2 & 2461191.750 & 155.750 & 17990 \\
10 & 121.2 & 2460970.594 & 155.750 & 18604 \\
20 & 121.1 & 2460872.057 & 155.750 & 18638 \\
30 & 121.2 & 2460863.037 & 155.750 & 18639 \\
50 & 121.2 & 2460857.512 & 155.750 & 18637 \\
75 & 121.9 & 2460852.957 & 155.750 & 18639 \\
100 & 121.8 & 2460850.886 & 155.750 & 18640 \\
125 & 121.8 & 2460848.991 & 155.750 & 18644 \\
150 & 121.8 & 2460847.192 & 155.750 & 18649 \\
\hline \hline
\end{tabular}

Table 2.1: Results for vertex-based procedure L1FIT1

point in $S$ which was the first point in the data structure of the implementation that was an endpoint of an edge in $T$ for which $F(i)$ was the other endpoint.

In all tables, the first column gives the sequence number of the particular pass. The second column displays its CPU running time in seconds. The third column contains the value of the objective function at the completion of the pass. Where applicable (Table 2.3), the fourth column contains the value of the residual tolerance used during the pass. The fifth column contains the maximum of the absolute values of the residuals at the completion of the pass. The last column contains the number of sharp grid points at the completion of the pass, i.e. the number of residuals whose absolute values were less than 0.01 at the completion of the pass.

We note that the numerical results in Table 2.1, Table 2.2, and Table 2.3 seem to indicate that the edge-based procedure works better than the vertex-based procedure, and that the $L_{2}$ with weights procedure works better than the edge-based procedure. However, the results in Table 2.4 seem to indicate that using the edge-based procedure after the execution of the $L_{2}$ with weights procedure is a better approach. 


\begin{tabular}{||r|ccccc||}
\hline \hline pass & CPUsec & objective & residual tolerance & MAX residual & \# sharp points \\
\hline 1 & 558.4 & 2476235.062 & 158.750 & 15552 \\
2 & 566.4 & 2460725.514 & 155.750 & 16867 \\
5 & 555.0 & 2457409.175 & 155.750 & 18986 \\
10 & 554.6 & 2457036.057 & 155.750 & 19573 \\
20 & 558.8 & 2457001.234 & 155.750 & 19683 \\
30 & 554.9 & 2456996.365 & 155.750 & 19649 \\
50 & 561.0 & 2456984.264 & 155.750 & 19595 \\
75 & 554.5 & 2456977.839 & 155.750 & 19552 \\
100 & 551.4 & 2456973.258 & 155.750 & 19536 \\
125 & 558.9 & 2456969.810 & 155.750 & 19505 \\
150 & 552.5 & 2456966.380 & 155.750 & 19494 \\
\hline \hline
\end{tabular}

Table 2.2: Results for edge-based procedure L1FIT2

\begin{tabular}{||r|ccccc||}
\hline \hline pass & CPUsec & objective & residual tolerance & MAX residual & \# sharp points \\
\hline 1 & 121.5 & 2588582.203 & 146.825 & 121.619 & 268 \\
5 & 121.5 & 2557018.183 & 120.793 & 126.196 & 280 \\
10 & 122.3 & 2556963.799 & 94.645 & 129.640 & 278 \\
20 & 122.1 & 2554668.834 & 58.104 & 135.601 & 282 \\
30 & 122.1 & 2542027.536 & 35.671 & 137.130 & 279 \\
50 & 122.0 & 2499697.025 & 13.444 & 142.532 & 262 \\
75 & 122.0 & 2469877.300 & 3.970 & 150.890 & 321 \\
100 & 121.9 & 2460298.750 & 1.172 & 154.523 & 446 \\
125 & 121.9 & 2457317.776 & 0.346 & 155.564 & 900 \\
150 & 121.9 & 2456400.229 & $1.022 \mathrm{e}-01$ & 155.702 & 2117 \\
175 & 121.9 & 2456122.316 & $3.019 \mathrm{e}-02$ & 155.737 & 6701 \\
200 & 121.9 & 2456038.092 & $8.915 \mathrm{e}-03$ & 155.745 & 18046 \\
225 & 121.9 & 2456015.632 & $2.633 \mathrm{e}-03$ & 155.749 & 18589 \\
250 & 121.9 & 2456013.435 & $7.774 \mathrm{e}-04$ & 155.749 & 18631 \\
275 & 123.1 & 2456013.310 & $6.058 \mathrm{e}-04$ & 155.749 & 18603 \\
300 & 122.2 & 2456013.128 & $6.058 \mathrm{e}-04$ & 155.749 & 18584 \\
\hline \hline
\end{tabular}

Table 2.3: Results for $L_{2}$-based procedure L1FIT3 


\begin{tabular}{||c|ccccc|}
\hline \hline pass & CPUsec & objective & residual tolerance & MAX residual & \# sharp points \\
\hline 1 & 545.8 & 2455998.827 & 155.748 & 19161 \\
2 & 553.8 & 2455994.372 & 155.748 & 19318 \\
5 & 537.3 & 2455988.608 & 155.750 & 19545 \\
10 & 539.4 & 2455987.781 & 155.750 & 19580 \\
20 & 539.8 & 2455987.481 & 155.750 & 19576 \\
30 & 539.9 & 2455987.377 & 155.750 & 19578 \\
50 & 547.7 & 2455987.331 & 155.750 & 19570 \\
70 & 540.8 & 2455987.276 & 155.750 & 19569 \\
150 & 539.7 & 2455987.265 & 155.750 & 19558 \\
\hline \hline
\end{tabular}

Table 2.4: Results for edge-based L1FIT2 after L1FIT3

\section{The 2-dimensional $L_{1}$ fitting problem}

Let $R$ be a regular grid with rectangular cell units covering a rectangular map in the plane. Let $S$ be a finite set of critical points in the map that contains the four corners of the map, and let $T$ be a triangulation for $S$. Let $S^{\prime}$ be the set of feature points, that is, of critical points with prescribed fixed elevations. For each grid point $p$ in $R$ assume that an elevation $\hat{z}_{p}$ is associated with $p$. Given $p$ in $R$, let $t$ be a triangle in $T$ that contains $p$, and define $s_{i}(p), i=1,2,3$, as the points in $S$ that are the vertices of $t$. Given $p$ in $R$, define $\lambda_{i}(p)$, $i=1,2,3$, as the barycentric coordinates of $p$ relative to $T$ so that $p$ equals $\sum_{i=1}^{3} \lambda_{i}(p) s_{i}(p)$ and $0 \leq \lambda_{i}(p) \leq 1$ for each $i, i=1,2,3$. For each point $s$ in $S^{\prime}$ assume that an elevation $z_{s}$ is associated with $s$. We search then for a set of elevations $\left\{z_{s}: s \in S \backslash S^{\prime}\right\}$ so that the following function is minimized:

$$
\sum_{p \in R}\left|\hat{z}_{p}-\sum_{i=1}^{3} \lambda_{i}(p) z_{s_{i}(p)}\right| .
$$

More generally, given $u, v>0$, and if for each $p$ in $R$ we let $\bar{z}_{p}$ equal $\sum_{i=1}^{3} \lambda_{i}(p) z_{s_{i}(p)}$, we search then for a set of elevations $\left\{z_{s}: s \in S \backslash S^{\prime}\right\}$ so that the following function is minimized:

$$
u \sum_{p \in R^{+}}\left(\hat{z}_{p}-\bar{z}_{p}\right)-v \sum_{p \in R^{-}}\left(\hat{z}_{p}-\bar{z}_{p}\right),
$$

where $R^{+}=\left\{p \in R: \hat{z}_{p}-\bar{z}_{p} \geq 0\right\}$ and $R^{-}=\left\{p \in R: \hat{z}_{p}-\bar{z}_{p}<0\right\}$. 


\section{The 2-dimensional $L_{2}$ fitting problem}

Of related interest is the quadratic version of the problem. Here we search for a set of elevations $\left\{z_{s}: s \in S \backslash S^{\prime}\right\}$ so that the following function is minimized:

$$
\sum_{p \in R}\left(\hat{z}_{p}-\sum_{i=1}^{3} \lambda_{i}(p) z_{s_{i}(p)}\right)^{2}
$$

Since this function is quadratic it has a unique minimum point which is the point at which its gradient equals zero [2].

Given a point $s$ in $S$ we define the star of $s$ as the union of the triangles in $T$ that have $s$ as a vertex, and denote by $R_{s}$ the set of grid points that it contains.

For a fixed point $s$ in $S$, we assume without any loss of generality that for each point $p$ in $R_{s}, s_{1}(p)$ equals $s$. Under this assumption the partial derivative of Function 4.1 with respect to $z_{s}$ is

$$
2 \sum_{p \in R_{s}}\left(\hat{z}_{p}-\lambda_{\mathbf{l}}(p) z_{s}-\lambda_{2}(p) z_{s_{2}(p)}-\lambda_{3}(p) z_{s_{3}(p)}\right)\left(-\lambda_{1}(p)\right) .
$$

Setting this partial derivative to zero we are then able to solve for $z_{s}$ and obtain

$$
z_{s}=\left(\sum_{p \in R_{s}}\left(\hat{z}_{p}-\lambda_{2}(p) z_{s_{2}(p)}-\lambda_{3}(p) z_{s_{3}(p)}\right) \lambda_{1}(p)\right) / \sum_{p \in R_{s}}\left(\lambda_{\mathbf{l}}(p)\right)^{2} .
$$

The following iterative procedure, which is based on the above formula, can be used for computing the minimum point of Function 4.1. That it terminates in a finite number of steps follows again from the fact that Function 4.1 is quadratic [2]. Here we assume that there are $n$ points in $S, n$ a positive integer, and that the $n$ points are ordered in some fashion. A one-to-one function $F$ from $\{1, \ldots, n\}$ onto $S$ is defined by setting $F(i)$ to the $i^{\text {th }}$ point in $S$ for each $i, i=1, \ldots, n$. A real-valued elevation function $Z$ with domain $S$ is defined by setting $Z(s)$ to $z_{s}$ for each $s$ in $S^{\prime}$, and to $\hat{z}_{s}$ otherwise. At the end of the execution of the procedure $Z$ will contain the minimum point. Another real-valued elevation function $\hat{Z}$ with domain $R$ is defined by setting $\hat{Z}(p)$ to $\hat{z}_{p}$ for each $p$ in $R$. The procedure, called L2FIT, requires an input variable called tol which must be set to a positive number and is used as a tolerance or adjustment constant during the execution of the procedure. Two procedures, called STARGRIDPOINTS and BARYCENTRIC are used as primitives in L2FIT. Given integer $i, 1 \leq i \leq n$, with $F(i) \in S \backslash S^{\prime}$, for some positive integer $m$, STARGRIDPOINTS locates points $G(j), j=1, \ldots, m$, that are the points in $R_{F(i)}$. Given an integer $j, 1 \leq j \leq m$, BARYCENTRIC locates a triangle in $T$ with $F(i)$ as a vertex that contains $G(j)$, identifies the other two vertices $Q_{2}, Q_{3}$ of the triangle, and computes the barycentric coordinates $W_{1}$, $W_{2}, W_{3}$ of $G(j)$ relative to the triangle so that $G(j)$ equals $W_{1} \cdot F(i)+W_{2} \cdot Q_{2}+W_{3} \cdot Q_{3}$. The outline of the procedure follows. 


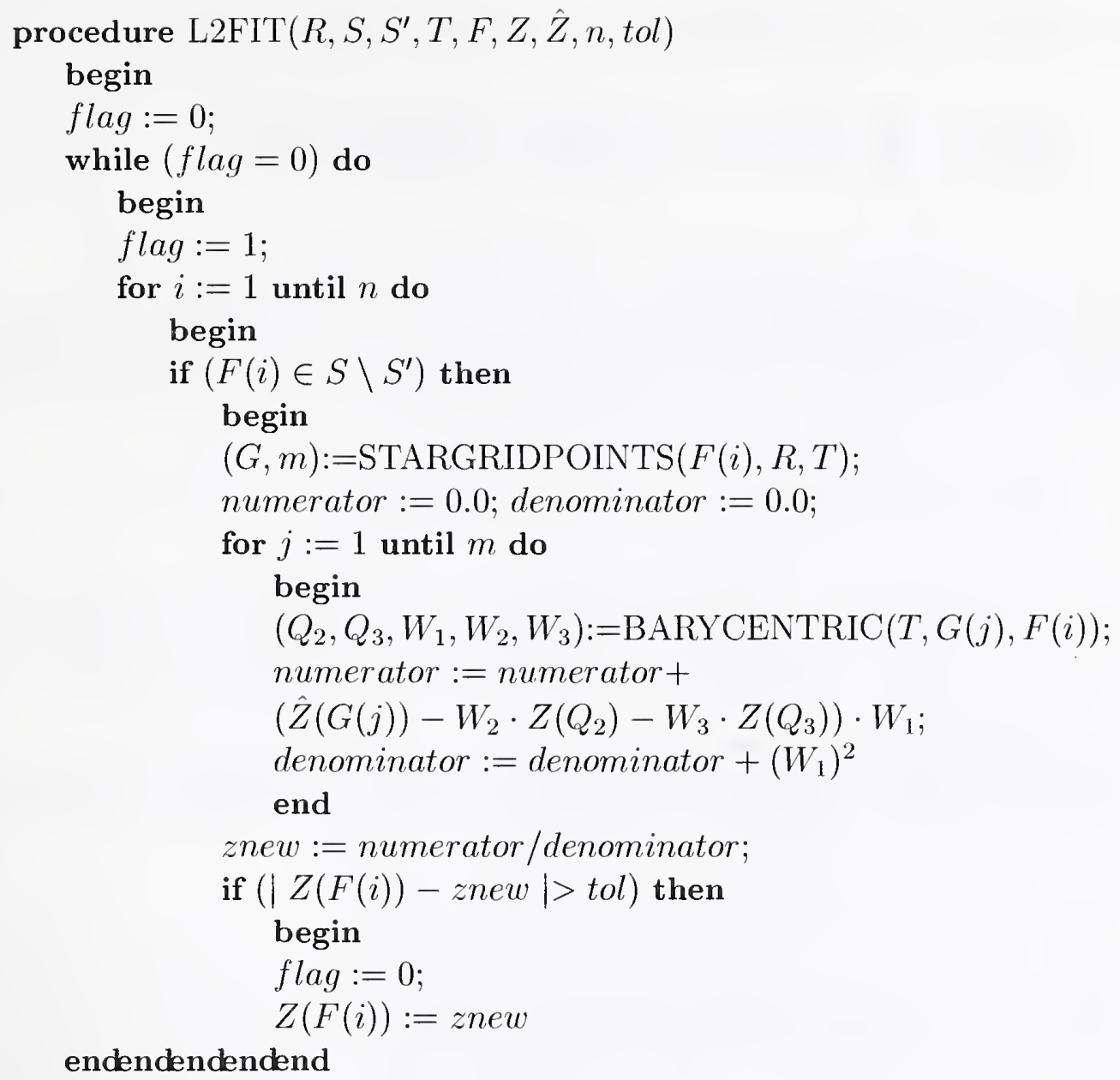

\section{Vertex-based iterative procedure for $L_{1}$ approximation}

An approach similar to the one in the previous section can be used for obtaining approximate solutions to $L_{1}$ fitting problems. Given $s$ in $S \backslash S^{\prime}$, for each point $\hat{s}$ in $S \backslash\{s\}$ we assume that an elevation $z_{\hat{s}}$ is associated with $\hat{s}$. We search then for an elevation $z_{s}$ so that the function

$$
\sum_{p \in R}\left|\hat{z}_{p}-\sum_{i=1}^{3} \lambda_{i}(p) z_{s_{i}(p)}\right|
$$

is minimized. 
More generally; given $u, v>0$, we search for an elevation $z_{s}$ so that the function

$$
u \sum_{p \in R^{+}}\left(\hat{z}_{p}-\sum_{i=1}^{3} \lambda_{i}(p) z_{s_{i}(p)}\right)-v \sum_{p \in R^{-}}\left(\hat{z}_{p}-\sum_{i=1}^{3} \lambda_{i}(p) z_{s_{i}(p)}\right)
$$

is minimized, where

$R^{+}=\left\{p \in R: \hat{z}_{p}-\sum_{i=1}^{3} \lambda_{i}(p) z_{s_{i}(p)} \geq 0\right\}$ and $R^{-}=\left\{p \in R: \hat{z}_{p}-\sum_{i=1}^{3} \lambda_{i}(p) z_{s_{i}(p)}<0\right\}$.

Assume without any loss of generality that for each point $p$ in $R_{s}, s_{1}(p)$ equals $s$, and for some positive integer $m$, let $p_{j}, j=1, \ldots, m$ be the points in $R_{s}$. The above problem is then equivalent to searching for an elevation $z_{s}$ so that the function

$$
\sum_{j=1}^{m} w_{j}\left|\hat{z}_{p_{j}}-\lambda_{1}\left(p_{j}\right) z_{s}-\lambda_{2}\left(p_{j}\right) z_{s_{2}\left(p_{j}\right)}-\lambda_{3}\left(p_{j}\right) z_{s_{3}\left(p_{j}\right)}\right|
$$

is minimized, where $w_{j}$ equals $u$ if $\hat{z}_{p_{j}}-\lambda_{1}\left(p_{j}\right) z_{s}-\lambda_{2}\left(p_{j}\right) z_{s_{2}\left(p_{j}\right)}-\lambda_{3}\left(p_{j}\right) z_{s_{3}\left(p_{j}\right)} \geq 0$, and $v$ otherwise, for each $j, j=1, \ldots, m$. This function is convex, continuous, piece-wise linear, and has at least one minimum point.

We show how a minimum point of Function 5.1 can be found. For each $j, j=1, \ldots, m$, assume without any loss of generality that $\lambda_{1}\left(p_{j}\right)$ is not equal to zero, and define $c_{j}$ as follows:

$$
c_{j} \equiv\left(\hat{z}_{p_{j}}-\lambda_{2}\left(p_{j}\right) z_{s_{2}\left(p_{j}\right)}-\lambda_{3}\left(p_{j}\right) z_{s_{3}\left(p_{j}\right)}\right) / \lambda_{1}\left(p_{j}\right) .
$$

Assume without any loss of generality that $m>1$, and that for each $j, j=1, \ldots, m-1$, $c_{j}<c_{j+1}$. For each $j, j=1, \ldots, m-1$, let $I_{j}$ be the interval $\left[c_{j}, c_{j+1}\right]$. Define $I_{0}$ as the interval $\left(-\infty, c_{1}\right]$, and $I_{m}$ as the interval $\left[c_{m}, \infty\right)$. In the interval $I_{0}$ the slope of Function 5.1 is $-u \sum_{k=1}^{m} \lambda_{1}\left(p_{k}\right)$, and in $I_{m}$ it is $v \sum_{k=1}^{m} \lambda_{1}\left(p_{k}\right)$. For each $j, j=1, \ldots, m-1$, in the interval $I_{j}$ the slope is $v \sum_{k=1}^{j} \lambda_{1}\left(p_{k}\right)-u \sum_{k=j+1}^{m} \lambda_{1}\left(p_{k}\right)$. Clearly, the slope must change signs at an endpoint of one the intervals, and this is then a minimum point of Function 5.1.

The following iterative procedure for attempting to minimize approximately Function 3.1, is based on the ideas presented above for minimizing Function 5.1. The procedure, called L1FIT1, requires an input variable called npmax which must be set to a positive integer and is used as the maximum number of passes allowed through the vertices, thus guaranteeing that the execution of the procedure terminates. Besides STARGRIDPOINTS and BARYCENTRIC, a procedure called INCREASORT is used as a primitive in L1FIT1. Given an integer $m w, m w>1$, a one-to-one function $M$ from $\{1, \ldots, m w\}$ onto a subset $I$ of the positive integers, and a function $C$ from $I$ into the set of real numbers, INCREASORT rearranges $M$ so that for each $j, j=1, \ldots, m w-1, C(M(j)) \leq C(M(j+1))$. The outline of the procedure follows. 


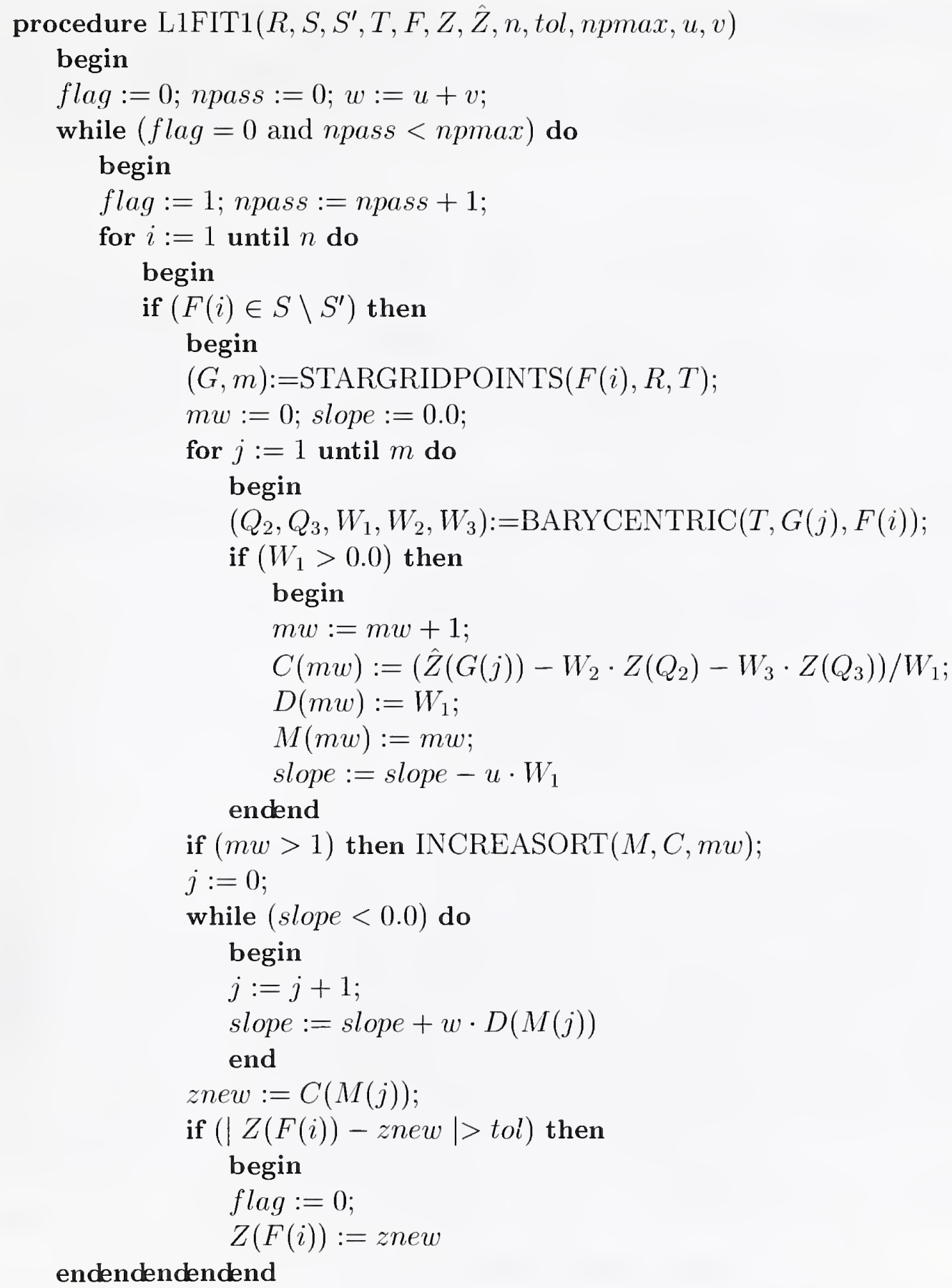




\section{Edge-based iterative procedure for $L_{1}$ approximation}

Givell $s, s^{\prime}$ in $S \backslash S^{\prime}, s$ and $s^{\prime}$ the endpoints of an edge of a triangle in $T$, and assuming that an elevation $z_{\hat{s}}$ is associated with each point $\hat{s}$ in $S \backslash\left\{s, s^{\prime}\right\}$, we now search for elevations $z_{s}$ and $z_{s^{\prime}}$ so that the function

$$
u \sum_{p \in R^{+}}\left(\hat{z}_{p}-\sum_{i=1}^{3} \lambda_{i}(p) z_{s_{i}(p)}\right)-v \sum_{p \in R^{-}}\left(\hat{z}_{p}-\sum_{i=1}^{3} \lambda_{i}(p) z_{s_{i}(p)}\right)
$$

is minimized. Here $R^{+}, R^{-}, u, v$ are as in the previous section.

Assume without any loss of generality that for each point $p$ in $R_{s}, s_{1}(p)$ equals $s$, that for each point $p$ in $R_{s^{\prime}}, s_{2}(p)$ equals $s^{\prime}$, and that the sets $R_{s} \backslash R_{s^{\prime}}$ and $R_{s^{\prime}} \backslash R_{s}$ are nonempty. For positive integers $m_{1}, m_{2}, m_{3}$, let $p_{j 1}, j=1, \ldots, m_{1}$ be the points in $R_{s} \cap R_{s^{\prime}}$, let $p_{j 2}$, $j=1, \ldots, m_{2}$ be the points in $R_{s} \backslash R_{s^{\prime}}$, and let $p_{j 3}, j=1, \ldots, m_{3}$ be the points in $R_{s^{\prime}} \backslash R_{s}$. The above problem is then equivalent to searching for elevations $z_{s}$ and $z_{s^{\prime}}$ so that the following function is minimized:

$$
\begin{aligned}
& \sum_{j=1}^{m_{1}} w_{1 j}\left|\hat{z}_{p_{j 1}}-\lambda_{1}\left(p_{j 1}\right) z_{s}-\lambda_{2}\left(p_{j 1}\right) z_{s^{\prime}}-\lambda_{3}\left(p_{j 1}\right) z_{s_{3}\left(p_{j 1}\right)}\right|+ \\
& \sum_{j=1}^{m_{2}} w_{2 j}\left|\hat{z}_{p_{j 2}}-\lambda_{1}\left(p_{j 2}\right) z_{s}-\lambda_{2}\left(p_{j 2}\right) z_{s_{2}\left(p_{j 2}\right)}-\lambda_{3}\left(p_{j 2}\right) z_{s_{3}\left(p_{j 2}\right)}\right|+ \\
& \sum_{j=1}^{m_{3}} w_{3 j}\left|\hat{z}_{p_{j 3}}-\lambda_{1}\left(p_{j 3}\right) z_{s_{1}\left(p_{j 3}\right)}-\lambda_{2}\left(p_{j 3}\right) z_{s^{\prime}}-\lambda_{3}\left(p_{j 3}\right) z_{s_{3}\left(p_{j 3}\right)}\right| .
\end{aligned}
$$

Here $w_{k j}$ equals $u$ if $\hat{z}_{p_{j k}}-\lambda_{1}\left(p_{j k}\right) z_{s_{1}\left(p_{j k}\right)}-\lambda_{2}\left(p_{j k}\right) z_{s_{2}\left(p_{j k}\right)}-\lambda_{3}\left(p_{j k}\right) z_{s_{3}\left(p_{j k}\right)} \geq 0$, and $v$ otherwise, for each $k, j, k=1,2,3, j=1, \ldots, m_{k}$. In what follows we think of Function 6.1 as a twovariable real-valued function whose first variable is $z_{s^{\prime}}$ and whose second variable is $z_{s}$. We denote it by $f$ so that if $z_{s}$ and $z_{s^{\prime}}$ are set to values, $f\left(z_{s^{\prime}}, z_{s}\right)$ then denotes the corresponding value of the function. Finally, we denote by $H\left(s^{\prime}, s\right)$ the set of triplets of the form $\left(z_{s^{\prime}}, z_{s}, f\left(z_{s^{\prime}}, z_{s}\right)\right)$. $H\left(s^{\prime}, s\right)$ is a convex, continuous, piece-wise linear surface in 3-dimensional space, and $z_{s}, z_{s^{\prime}}$ exist at which $f$ achieves its minimum value, and $\left(z_{s^{\prime}}, z_{s}, f\left(z_{s^{\prime}}, z_{s}\right)\right)$ is a vertex of $H\left(s^{\prime}, s\right)$.

For each $j, j=1, \ldots, m_{1}$, assume without any loss of generality that $\lambda_{1}\left(p_{j 1}\right)$ is not equal to zero, and define $c_{j}, b_{j}, d_{j}$ as follows:

$$
\begin{aligned}
c_{j} & \equiv\left(\hat{z}_{p_{j 1}}-\lambda_{3}\left(p_{j 1}\right) z_{s_{3}\left(p_{j 1}\right)}\right) / \lambda_{1}\left(p_{j 1}\right), \\
b_{j} & \equiv-\lambda_{2}\left(p_{j 1}\right) / \lambda_{1}\left(p_{j 1}\right), \\
d_{j} & \equiv \lambda_{1}\left(p_{j 1}\right) .
\end{aligned}
$$


For each $j, j=1, \ldots, m_{2}$, assume without any loss of generality that $\lambda_{1}\left(p_{j 2}\right)$ is not equal to zero, and define $c_{j+m_{1}}, b_{j+m_{1}}, d_{j+m_{1}}$ as follows:

$$
\begin{aligned}
c_{j+m_{1}} & \equiv\left(\hat{z}_{p_{j 2}}-\lambda_{2}\left(p_{j 2}\right) z_{s_{2}\left(p_{j 2}\right)}-\lambda_{3}\left(p_{j 2}\right) z_{s_{3}\left(p_{j 2}\right)}\right) / \lambda_{1}\left(p_{j 2}\right), \\
b_{j+m_{1}} & \equiv 0.0 \\
d_{j+m_{1}} & \equiv \lambda_{1}\left(p_{j 2}\right) .
\end{aligned}
$$

For each $j, j=1, \ldots, m_{3}$, assume without any loss of generality that $\lambda_{2}\left(p_{j 3}\right)$ is not equal to zero, and define $c_{j}^{\prime}, d_{j}^{\prime}$ as follows:

$$
\begin{aligned}
c_{j}^{\prime} & \equiv\left(\hat{z}_{p_{j 3}}-\lambda_{1}\left(p_{j 3}\right) z_{s_{1}\left(p_{j 3}\right)}-\lambda_{3}\left(p_{j 3}\right) z_{s_{3}\left(p_{j 3}\right)}\right) / \lambda_{2}\left(p_{j 3}\right), \\
d_{j}^{\prime} & \equiv \lambda_{2}\left(p_{j 3}\right) .
\end{aligned}
$$

Setting $m w$ to $m_{1}+m_{2}$, and $m v$ to $m_{3}$, for each $j, j=1, \ldots, m w$, denote by $l_{j}$ the straight line in the $z_{s^{\prime}}-z_{s}$ plane defined by the linear equation $z_{s}=b_{j} z_{s^{\prime}}+c_{j}$, and for each $j$, $j=1, \ldots, m v$, denote by $l_{j}^{\prime}$ the straight line in the same plane defined by the linear equation $z_{s}^{\prime}=c_{j}^{\prime}$. Clearly, from the polyhedral nature of $H\left(s^{\prime}, s\right)$ it follows that the perpendicular projections onto the $z_{s^{\prime}}-z_{s}$ plane of the 1-dimensional faces of $H\left(s^{\prime}, s\right)$ are contained in these lines, and those of the vertices are points where these lines intersect. Thus, the problem of minimizing Function 6.1 reduces to that of finding among the points at which at least two of the lines $l_{j}, j=1, \ldots, m w, l_{j}^{\prime}, j=1, \ldots, m v$, intersect, one at which the function achieves its smallest value.

Given an integer mopt, $1 \leq m o p t \leq m w$, we show how a minimum point of $f$, i. e. Function 6.1 , restricted to line $l_{\text {mopt }}$ can be found in a manner similar to the one used in the previous section for minimizing Function 5.1. Without any loss of generality we assume that at any point in the $z_{s^{\prime}}-z_{s}$ plane at most two of the lines $l_{j}, j=1, \ldots, m w, l_{j}^{\prime}, j=1, \ldots, m v$, intersect. Thus, if two of these lines intersect, and Function 6.1 restricted to the two lines achieves its smallest value at the point at which the two lines intersect, then from the convexity and the polyhedral nature of $H\left(s^{\prime}, s\right)$ it follows that anong the points at which any two of the lines intersect, at this point the function achieves its smallest value. Therefore, as pointed above, this then implies that at this point the function also achieves its minimum value without any restrictions on $z_{s}$ and $z_{s^{\prime}}$.

For each $j, j=1, \ldots, m w, j \neq m o p t$, assume without any loss of generality that $l_{j}$ is not parallel to $l_{\text {mopt }}$, and define $a_{j}$ as follows:

$$
a_{j} \equiv\left(c_{j}-c_{m o p t}\right) /\left(b_{m o p t}-b_{j}\right)
$$

Clearly, $a_{j}$ is the value of $z_{s^{\prime}}$ at which $l_{j}$ intersects $l_{\text {mopt }}$. Assume without any loss of generality that $m w>2$, that mopt equals $m w$, and that for each $j, j=1, \ldots, m w-2$, 
$a_{j}<a_{j+1}$. For the sake of simplicity and only for the current discussion, assume that $m v$ equals zero, and for each $j, j=1, \ldots, m w-2$, let $I_{j}$ be the interval $\left[a_{j}, a_{j+1}\right]$ in the $z_{s^{\prime}}$ axis of the $z_{s^{\prime}}-z_{s}$ plane. Define $I_{0}$ as the interval $\left(-\infty, a_{1}\right]$, and $I_{m w-1}$ as the interval $\left[a_{m w-1}, \infty\right)$ in the same axis. Define a one-variable real-valued function $f^{\prime}$ by setting $f^{\prime}\left(z_{s^{\prime}}\right)$ to $f\left(z_{s^{\prime}}, b_{\text {mopt }} z_{s^{\prime}}+c_{\text {mopt }}\right)$ for each value of $z_{s^{\prime}}$. $f^{\prime}$ is convex, continuous, piece-wise linear, and has at least one minimum point. For each $j$, set $u_{j}$ to $u$ if $b_{m o p t}-b_{j}$ is positive, and to $-v$ otherwise, and set $v_{j}$ to $v$ if $b_{m o p t}-b_{j}$ is positive, and to $-u$ otherwise. With this terminology, in the interval $I_{0}$ the slope of $f^{\prime}$ is $-\sum_{k=1}^{m w-1} u_{j}\left(b_{m o p t}-b_{j}\right) d_{j}$, and in $I_{m w-1}$ it is $\sum_{k=1}^{m w-1} v_{j}\left(b_{m o p t}-b_{j}\right) d_{j}$. For each $j, j=1, \ldots, m w-2$, in the interval $I_{j}$ the slope is $\sum_{k=1}^{j} v_{j}\left(b_{\text {mopt }}-b_{j}\right) d_{j}-\sum_{k=j+1}^{m w-1} u_{j}\left(b_{m o p t}-b_{j}\right) d_{j}$. Clearly, the slope of $f^{\prime}$ must change signs at an endpoint of one of the intervals, and this is then a minimum point of $f^{\prime}$. Thus, given $z_{s^{\prime}}$ at which $f^{\prime}$ achieves its minimum value it follows that Function 6.1 restricted to $l_{\text {mopt }}$ has a minimum point at $\left(z_{s^{\prime}}, b_{\text {mopt }} z_{s^{\prime}}+c_{\text {mopt }}\right)$.

A minimum point of Function 6.1 without any restrictions on $z_{s}$ and $z_{s^{\prime}}$ can then be found through a two-step iterative procedure as follows:

Step 1. Mark each one of the lines $l_{j}, j=1, \ldots, m w, l_{j}^{\prime}, j=1, \ldots, m v$ as not optimized. Select arbitrarily one of these lines and call it the current line.

Step 2. Find a minimum point of Function 6.1 restricted to the current line, and call this point the current minimum point. Mark the current line as optimized, identify the one line among the lines $l_{j}, j=1, \ldots, m w, l_{j}^{\prime}, j=1, \ldots, m v$, that intersects the current line at the current minimum point, and call this line the next line. If the next line has been marked as optimized then the current minimum point is a minimum point of Function 6.1 without restrictions and the procedure terminates. Else call the next line the current line and go back to the beginning of Step 2.

The following iterative procedure for attempting to minimize approximately Function 3.1, is based on the ideas presented above for minimizing Function 6.1. Here we assume that for each $i, i=1, \ldots, n$, an edge of a triangle in $T$ is selected so that $F(i)$ is an endpoint of this edge. A function $E$ from $\{1, \ldots, n\}$ into $S$ is defined by setting for each $i, i=1, \ldots, n$, $E(i)$ to the point in $S$ which is the other endpoint of the edge selected with $F(i)$ as an endpoint. Besides INCREASORT, the procedure, called L1FIT2, requires primitives STARGRIDPOINTS1, STARGRIDPOINTS2, STARGRIDPOINTS3 as well as BARYCENTRIC1, BARYCENTRIC2, and BARYCENTRIC3. Given integer $i, 1 \leq i \leq n$, with $F(i) \in S \backslash S^{\prime}$, for some positive integer $m_{1}$, STARGRIDPOINTS1 locates points $G_{1}(j), j=1, \ldots, m_{1}$, that are the points in $R_{F(i)} \cap R_{E(i)}$. Given that $R_{F(i)} \backslash R_{E(i)}$ is not empty, for some positive integer $m_{2}$, STARGRIDPOINTS2 locates points $G_{2}(j), j=1, \ldots, m_{2}$, that are the points in $R_{F(i)} \backslash R_{E(i)}$. It sets $m_{2}$ to zero if $R_{F(i)} \backslash R_{E(i)}$ is empty. Given that $R_{E(i)} \backslash R_{F(i)}$ is not empty, for some positive integer $m_{3}$, STARGRIDPOINTS3 locates points $G_{3}(j), j=1, \ldots, m_{3}$, that are the points 
in $R_{E(i)} \backslash R_{F(i)}$. It sets $m_{3}$ to zero if $R_{E(i)} \backslash R_{F(i)}$ is empty. Given an integer $j, 1 \leq j \leq m_{1}$, BARYCENTRIC1 locates a triangle in $T$ with $F(i)$ and $E(i)$ as vertices that contains $G_{1}(j)$, identifies the third vertex $Q_{3}$ of the triangle, and computes the barycentric coordinates $W_{1}$, $W_{2}, W_{3}$ of $G_{1}(j)$ relative to the triangle so that $G_{1}(j)$ equals $W_{1} \cdot F(i)+W_{2} \cdot E(i)+W_{3} \cdot Q_{3}$. Assuming $m_{2}$ is positive, given an integer $j, 1 \leq j \leq m_{2}$, BARYCENTRIC2 locates a triangle in $T$ with $F(i)$ as a vertex that contains $G_{2}(j)$, identifies the other two vertices $Q_{2}, Q_{3}$ of the triangle, and computes the barycentric coordinates $W_{1}, W_{2}, W_{3}$ of $G_{2}(j)$ relative to the triangle so that $G_{2}(j)$ equals $W_{1} \cdot F(i)+W_{2} \cdot Q_{2}+W_{3} \cdot Q_{3}$. Finally, assuming $m_{3}$ is positive, given an integer $j, 1 \leq j \leq m_{3}$, BARYCENTRIC3 locates a triangle in $T$ with $E(i)$ as a vertex that contains $G_{3}(j)$, identifies the other two vertices $Q_{1}, Q_{3}$ of the triangle, and computes the barycentric coordinates $W_{1}, W_{2}, W_{3}$ of $G_{3}(j)$ relative to the triangle so that $G_{3}(j)$ equals $W_{1} \cdot Q_{1}+W_{2} \cdot E(i)+W_{3} \cdot Q_{3}$.

The outline of the procedure follows. In lines 6-41 of the procedure, the nonvertical straight lines $\left(l_{j}, j=1, \ldots, m w\right.$, above $)$, and the vertical straight lines $\left(l_{j}^{\prime}, j=1, \ldots, m v\right.$, above) associated with the current edge are identified. In lines 42-60 of the procedure the first straight line to be called the current line (for the current edge) is identified, and it is the one nonvertical line at which Function 6.1 (for the current edge) attains its minimum for $z_{s^{\prime}}$ arbitrarily close to $-\infty$. In line 66 the $z_{s^{\prime}}$ values where the vertical lines intersect the $z_{s^{\prime}}$-axis are sorted in increasing order. The $z_{s^{\prime}}$ values where the current line intersects nonvertical lines are computed in lines $72-82$ of the procedure if the current line is nonvertical, and in lines 111-114 if it is vertical. These values are sorted in increasing order in line 84 if the current line is nonvertical, and in line 115 if it is vertical. In lines 87-99 if the current line is nonvertical, and in lines 117-120 if it is vertical, a straight line to be called the next line is identified which is either a nonvertical or a vertical line whose intersection with the current line is a minimum point of Function 6.1 restricted to the current line (to be called the current minimum point). The current line is marked as optimized in line 106 of the procedure if it is nonvertical and in line 109 if it is vertical. Given that the next line has already been marked as optimized, in lines 122-128 of the procedure if the current line is vertical, and in lines 129-134 if it is nonvertical, another nonvertical line, if any, to be called the current line is identified which is not marked as optimized and that contains the current minimum point. If no such a line exists then the current minimum point is a minimum point for Function 6.1 without restrictions and if necessary the values of $z_{s}$ and $z_{s^{\prime}}$ (for the current edge) are corrected in lines 135-140 of the procedure.

procedure L1FIT2 $\left(R, S, S^{\prime}, T, F, E, Z, \hat{Z}, n\right.$, tol, npmax $\left., u, v\right)$ begin

1. $\quad f l a g 1:=0 ;$ npass $:=0 ; w:=u+v$;

2. while $($ flag $1=0$ and npass $<$ npmax $)$ do

begin 


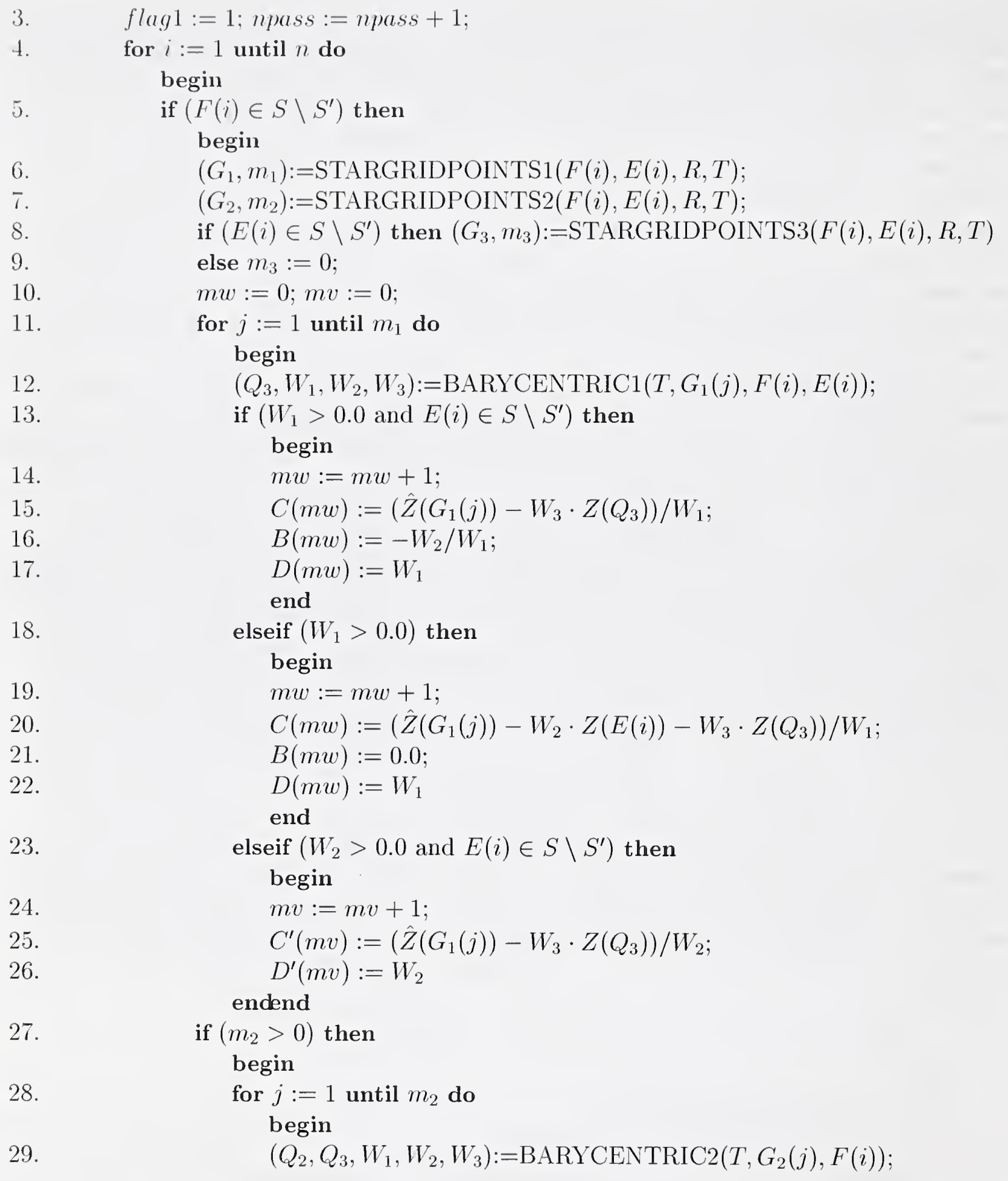


30.

31.

32.

33.

34.

35.

36.

37.

38.

39.

40.

41.

42.

43.

44.

45.

46.

47.

48.

49.

50.

51.

52.

53.

54.

if $\left(W_{1}>0.0\right)$ then

\section{begin}

$$
m w:=m w+1 \text {; }
$$

$C(m w):=\left(\hat{Z}\left(G_{2}(j)\right)-W_{2} \cdot Z\left(Q_{2}\right)-W_{3} \cdot Z\left(Q_{3}\right)\right) / W_{1} ;$

$B(m w):=0.0$

$$
D(m w):=W_{1}
$$

\section{endendend}

if $\left(m_{3}>0\right)$ then

begin

for $j:=1$ until $m_{3}$ do

begin

$\left(Q_{1}, Q_{3}, W_{1}, W_{2}, W_{3}\right):=\operatorname{BARYCENTRIC} 3\left(T, G_{3}(j), E(i)\right)$; if $\left(W_{2}>0.0\right)$ then

\section{begin}

$$
\begin{aligned}
& m v:=m v+1 ; \\
& C^{\prime}(m v):=\left(\hat{Z}\left(G_{3}(j)\right)-W_{1} \cdot Z\left(Q_{1}\right)-W_{3} \cdot Z\left(Q_{3}\right)\right) / W_{2} ;
\end{aligned}
$$

$$
D^{\prime}(m v):=W_{2}
$$

endendend

slopew $:=0.0$;

for $j:=1$ until $m w$ do

begin

$M(j):=j ; N(j):=0$

slopew $:=$ slopew $+v \cdot D(j)$

end

if $(m w>1)$ then

begin

INCREASORT $(M, B, m w)$;

for $j:=1$ until $m w-1$ do

begin

$$
j j:=j+1 \text {; }
$$

while $(j j \leq m w$ and $B(M(j j))=B(M(j)))$ do

$$
\text { begin }
$$$$
\text { if }(C(M(j j))>C(M(j))) \text { then }
$$

$$
\begin{aligned}
& \text { begin } \\
& m t:=M(j) ;
\end{aligned}
$$

$M(j):=M(j j)$;

$M(j j):=m t$

end 
$5 \overline{5}$

56.

57.

58.

59.

60.

61.

62.

63.

64.

65.

66.

67.

68.

69.

70.

71.

72.

73.

74.

75.

76.

77.

78.

79.

80.

81.

82.

83.

$$
j j:=j j+1
$$

endendend

$$
\begin{aligned}
& j:=0 ; \\
& \text { while }(\text { slopew }>0.0) \text { do } \\
& \quad \text { begin } \\
& j:=j+1 ; \\
& \quad \text { slopew }:=\text { slopew }-w \cdot D(M(j)) \\
& \quad \text { end }
\end{aligned}
$$

mopt $:=M(j)$;

slopev $:=0.0$;

if $(m v>0)$ then

$$
\text { begin }
$$

$$
\text { for } j:=1 \text { until } m v \text { do }
$$

\section{begin}

$$
\begin{aligned}
& M^{\prime}(j):=j ; N^{\prime}(j):=0 ; \\
& \text { slopev }:=\text { slopev }+u \cdot D^{\prime}(j)
\end{aligned}
$$

\section{endend}

if $(m v>1)$ then INCREASORT $\left(M^{\prime}, C^{\prime}, m v\right)$;

$z n w a:=C($ mopt $) ; z n w b:=Z(E(i))$;

flag $2:=0$;

while $(f \operatorname{lag} 2=0)$ do

\section{begin}

$$
\text { flag } 2:=1 ; m b:=0
$$

$m b c:=1 ; m v c:=1 ;$ slopew $:=$ slopev;

for $j:=1$ until $m w$ do

$$
\text { begin }
$$

$$
b:=B(\text { mopt })-B(j) \text {; }
$$

$$
\text { if }(b>0.0) \text { then }
$$

$$
\text { slopew }:=\text { slopew }+u \cdot b \cdot D(j)
$$

elseif $(b<0.0)$ then

$$
\text { slopew }:=\text { slopew }-v \cdot b \cdot D(j) ;
$$

if $(b \neq 0.0)$ then

begin

$$
\begin{aligned}
& c:=C(j)-C(\text { mopt }) \\
& m b:=m b+1 \\
& M(m b):=j \\
& A(j):=c / b
\end{aligned}
$$

endend

if $(m b>0$ or $m v>0)$ then 
84.

85.

86.

87.

88.

89.

90.

91.

92.

93.

94.

95.

96.

97.

98.

99.

100.

101.

102.

103.

104.

105.

106.

107.

108.

\section{begin}

if $(m b>1)$ then INCREASORT $(M, A, m b)$;

$M(m b+1):=m w+1 ; A(m w+1):=0.0 ;$

$M^{\prime}(m v+1):=m v+1 ; C^{\prime}(m v+1):=0.0$;

while $($ slopew $>0.0)$ do

begin

if $\left(\left(m b c \leq m b\right.\right.$ and $m v c \leq m v$ and $\left.A(M(m b c))<C^{\prime}\left(M^{\prime}(m v c)\right)\right)$ or $(m b c \leq m b$ and $m v c>m v))$ then

begin

flag $3:=1$;

$j b:=M(m b c)$;

$b:=B($ mopt $)-B(j b)$;

if $(b>0.0)$ then

$$
\text { slopew }:=\text { slopew }-w \cdot b \cdot D(j b)
$$

else

slopew $:=$ slopew $+w \cdot b \cdot D(j b) ;$

$m b c:=m b c+1$

end

else

\section{begin}

$f \operatorname{lag} 3:=2$;

$j v:=M^{\prime}(m v c)$

slopew $:=$ slopew $-w \cdot D^{\prime}(j v)$;

$m v c:=m v c+1$

endend

if $($ flag $3=1)$ then

begin

$z n w b:=A(j b)$;

if $(N(j b) \neq 0)$ then flag $3:=3$

end

else

begin

$z n w b:=C^{\prime}(j v)$

if $\left(N^{\prime}(j v) \neq 0\right)$ then flag $3:=4$

end

$z n w a:=B($ mopt $) \cdot z n w b+C($ mopt $)$;

$N($ mopt $):=1$;

if $(f \operatorname{lag} 3=1)$ then $\operatorname{mopt}:=j b ;$ flag $2:=0$

elseif $(f \operatorname{lag} 3=2)$ then 
109.

110.

111.

112.

113.

114.

115.

116.

117.

118.

119.

120.

121.

122.

123.

124.

125.

126.

127.

128.

129.

130.

131.

132.

133.

\section{begin}

$N^{\prime}(j v):=1$;

slopew $:=0.0$;

for $j:=1$ until $m w$ do

\section{begin}

$M(j):=j ;$

slopew $:=$ slopew $+u \cdot D(j)$;

$A(j):=B(j) \cdot z n w b+C(j)$

end

if $(m w>1)$ then $\operatorname{INCREASORT}(M, A, m w)$;

$j:=0$;

while $($ slopew $>0.0)$ do

begin

$j:=j+1$;

slopew $:=$ slopew $-w \cdot D(M(j))$

end

$j b:=M(j)$;

if $(N(j b)=0)$ then mopt $:=j b ; f \operatorname{lag} 2:=0$

else

begin

$z n w a:=A(j b)$;

$b:=0.0 ; k:=1 ; j j:=j+1$;

while $(b=0.0$ and $k=1$ and $j j \leq m w)$ do

begin

$b:=A(M(j j))-z n w a ;$

$k:=N(M(j j))$;

$j j:=j j+1$

end

if $(b=0.0$ and $k=0)$ then mopt $:=M(j j-1) ;$ flag $2:=0$ endend

else

begin

$b:=0.0 ; k:=1 ; j j:=m b c$;

while $(b=0.0$ and $k=1$ and $j j \leq m b)$ do

begin

$b:=A(M(j j))-z n w b$;

$k:=N(M(j j))$;

$j j:=j j+1$

end 
134.

135.

136.

137.

138.

139.

140.

if $(b=0.0$ and $k=0)$ then mopt $:=M(j j-1) ;$ flag $2:=0$ endendend
if $(|Z(F(i))-z n w a|>$ tol $)$ then
begin

\section{begin}

$\operatorname{flag} 1:=0$

$Z(F(i)):=$ znwa

end

if $(|Z(E(i))-z n w b|>t o l)$ then

begin

flag $1:=0$

endendendendend

$Z(E(i)):=z n w b$

\section{Residual-weighted $L_{2}$ iterative procedure for $L_{1}$ approximation}

An approach based on the $L_{2}$ iterative procedure presented in Section 4 can be used for obtaining approximate solutions to $L_{1}$ fitting problems. This is based on the fact that given a real number $r$ if $r$ does not equal zero then $|r|$ equals $r^{2} /|r|$.

Given $s$ in $S \backslash S^{\prime}$, we assume that an elevation $\tilde{z}$ and a positive number wcut are associated with $s$. In addition, for each point $\hat{s}$ in $S \backslash\{s\}$ we assume that an elevation $z_{\hat{s}}$ is associated with $\hat{s}$. Given $u, v>0$, and assuming again without any loss of generality that for each point $p$ in $R_{s}, s_{1}(p)$ equals $s$, and that for some positive integer $m, p_{j}, j=1, \ldots, m$, are the points in $R_{s}$, we search then for an elevation $z_{s}$ so that the function

$$
\sum_{j=1}^{m}\left(w_{j} / \tilde{w}_{j}\right)\left(\hat{z}_{p_{j}}-\lambda_{1}\left(p_{j}\right) z_{s}-\lambda_{2}\left(p_{j}\right) z_{s_{2}\left(p_{j}\right)}-\lambda_{3}\left(p_{j}\right) z_{s_{3}\left(p_{j}\right)}\right)^{2}
$$

is minimized. Here $w_{j}$ equals $u$ if $\hat{z}_{p_{j}}-\lambda_{1}\left(p_{j}\right) \tilde{z}-\lambda_{2}\left(p_{j}\right) z_{s_{2}\left(p_{j}\right)}-\lambda_{3}\left(p_{j}\right) z_{s_{3}\left(p_{j}\right)} \geq 0$, and $v$ otherwise; and $\tilde{w}_{j}$ equals $\left|\hat{z}_{p_{j}}-\lambda_{1}\left(p_{j}\right) \tilde{z}-\lambda_{2}\left(p_{j}\right) z_{s_{2}\left(p_{j}\right)}-\lambda_{3}\left(p_{j}\right) z_{s_{3}\left(p_{j}\right)}\right|$ if this last number is greater than wcut, and wcut otherwise. We notice that given $j, 1 \leq j \leq m$, if $\tilde{w}_{j}$ is greater than wcut and $z_{s}$ is set to $\tilde{z}$ then the $j^{t h}$ term of Function 7.1 becomes $\left(w_{j} / \tilde{w}_{j}\right) \tilde{w}_{j}^{2}$ which reduces to $w_{j} \tilde{w}_{j}$, i. e. $w_{j}\left|\hat{z}_{p_{j}}-\lambda_{1}\left(p_{j}\right) \tilde{z}-\lambda_{2}\left(p_{j}\right) z_{s_{2}\left(p_{j}\right)}-\lambda_{3}\left(p_{j}\right) z_{s_{3}\left(p_{j}\right)}\right|$.

The derivative of Function 7.1 with respect to $z_{s}$ is

$$
2 \sum_{j=1}^{m}\left(w_{j} / \tilde{w}_{j}\right)\left(\hat{z}_{p_{j}}-\lambda_{1}\left(p_{j}\right) z_{s}-\lambda_{2}\left(p_{j}\right) z_{s_{2}\left(p_{j}\right)}-\lambda_{3}\left(p_{j}\right) z_{s_{3}\left(p_{j}\right)}\right)\left(-\lambda_{1}\left(p_{j}\right)\right) .
$$

Setting this derivative to zero we are then able to solve for $z_{s}$ and thus obtain the value for 
$z_{s}$ at which Function 7.1 is minimized:

$$
z_{s}=\left(\sum_{j=1}^{m}\left(w_{j} / \tilde{w}_{j}\right)\left(\hat{z}_{p_{j}}-\lambda_{2}\left(p_{j}\right) z_{s_{2}\left(p_{j}\right)}-\lambda_{3}\left(p_{j}\right) z_{s_{3}\left(p_{j}\right)}\right) \lambda_{1}\left(p_{j}\right)\right) / \sum_{j=1}^{m}\left(w_{j} / \tilde{w}_{j}\right)\left(\lambda_{1}\left(p_{j}\right)\right)^{2} .
$$

The following iterative procedure, which is based on the above formula, can be used for attempting to minimize approximately Function 3.1. Besides STARGRIDPOINTS and BARYCENTRIC, the procedure, called L1FIT3, requires a primitive called MAXRESIDUAL. It computes wcut which is the largest absolute value of a residual, i. e. the largest absolute value of a number of the form $\hat{Z}(p)-W_{1} \cdot Z\left(Q_{1}\right)-W_{2} \cdot Z\left(Q_{2}\right)-W_{3} \cdot Z\left(Q_{3}\right)$, where $p$ is in $R, Q_{1}$, $Q_{2}, Q_{3}$ are the vertices of a triangle in $T$ that contains $p$, and $W_{1}, W_{2}, W_{3}$ are the barycentric coordinates of $p$ relative to the triangle so that $p$ equals $W_{1} \cdot Q_{1}+W_{2} \cdot Q_{2}+W_{3} \cdot Q_{3}$. The variable wcut, which we call the residual tolerance, is used throughout the execution of the procedure for the purpose of avoiding or postponing division by absolute values of residuals that are less than its current value. This value is progressively reduced by dividing wcut before each pass through the vertices by a variable called $w s c l$. This last variable must be set on input to a number that is greater than 1.0 and preferably less than or equal to 2.0. The outline of the procedure follows.

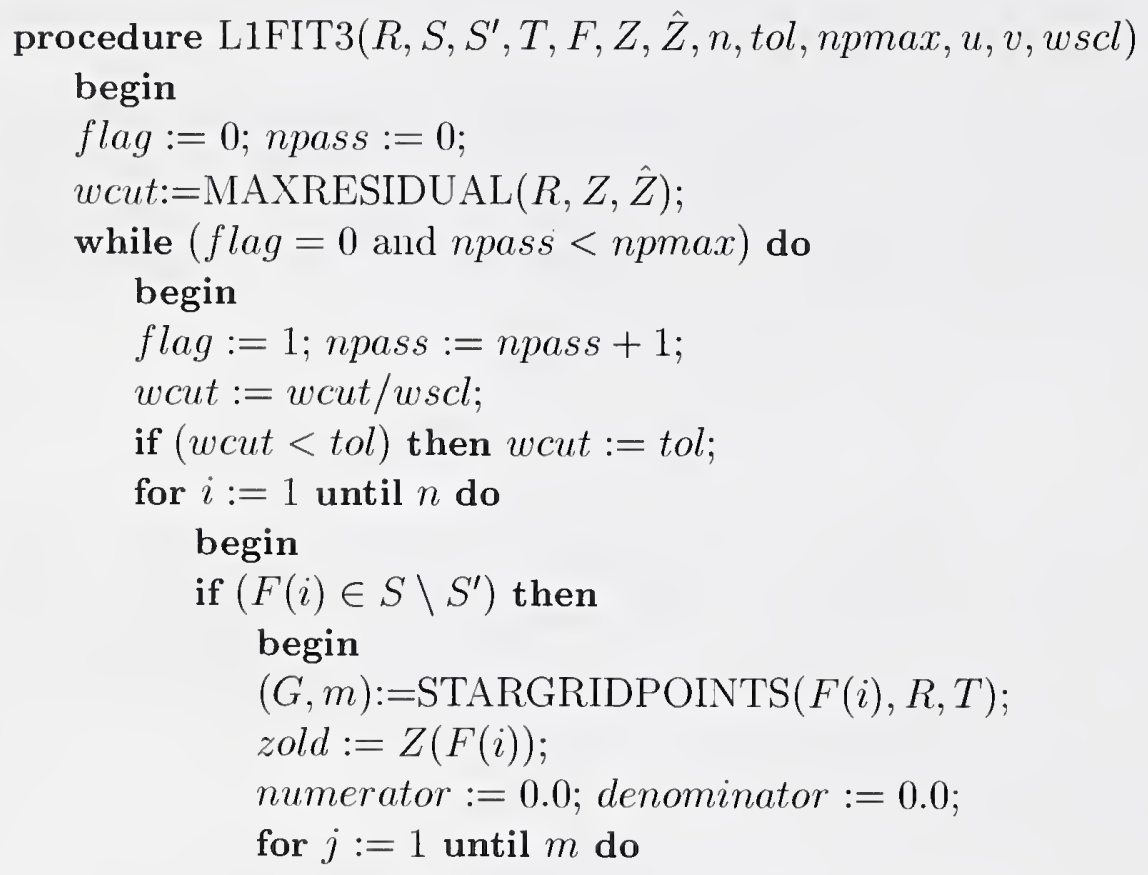

$$
\begin{aligned}
& \text { begin } \\
& \left(Q_{2}, Q_{3}, W_{1}, W_{2}, W_{3}\right):=\operatorname{BARYCENTRIC}(T, G(j), F(i)) \\
& \tilde{W}:=\hat{Z}(G(j))-W_{1} \cdot \text { zold }-W_{2} \cdot Z\left(Q_{2}\right)-W_{3} \cdot Z\left(Q_{3}\right) \\
& \text { if }(\tilde{W} \geq 0.0) \text { then } W:=u \text { else } W:=v
\end{aligned}
$$




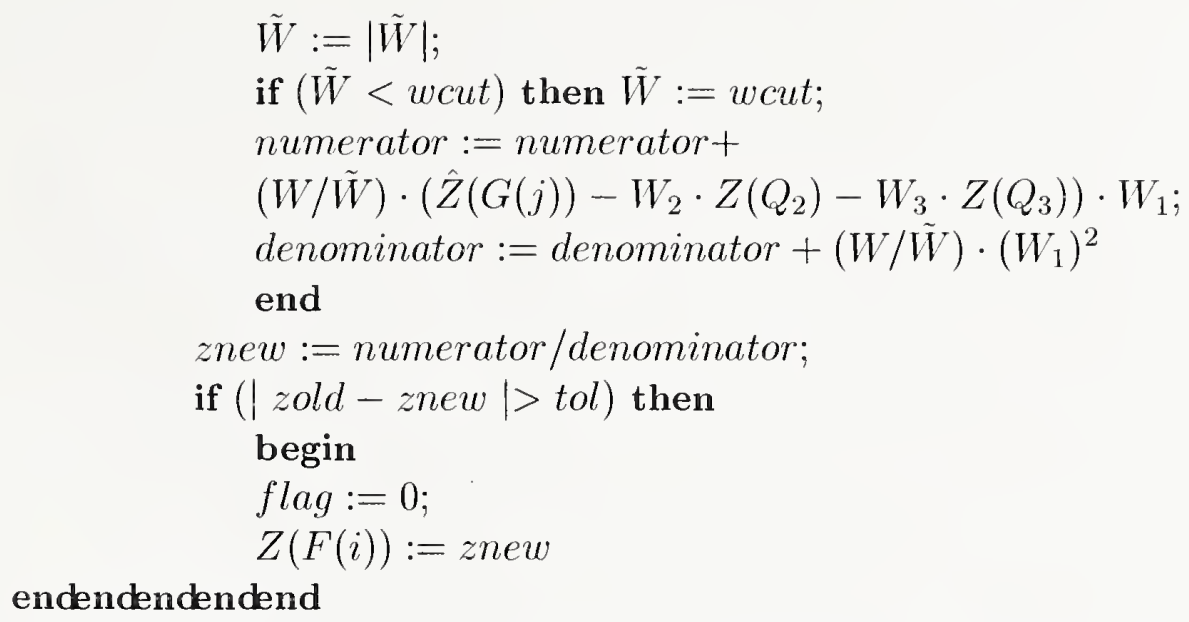

Acknowledgement. Thanks to Douglas R. Caldwell, Topographic Engineering Center (TEC), for his interest and support.

\section{BIBLIOGRAPHY}

[1] I.Barrodale and F. D. K. Roberts, An Improved Algorithm for Discrete $l_{1}$ Approximation, SIAM J. Numer. ANAL. 10 (1993), pp 839-848.

[2] D. G. Luenberger, Introduction to Linear and Nonlinear Programming, Addison-Wesley, Reading, Mass. (1973).

[3] D. M. Mark, The History of Geographic Information Systems: Invention and Re-invention of Triangulated Irregular Networks (TINs), Proceedings GIS/LIS'97, in press.

(http://www.geog.buffalo.edu/ dmark/)

[4] C. Witzgall and D. R. Caldwell, An $L_{1}$-Optimized TIN Approach to Locating Terrain Data Artifacts. (In preparation) 


